\title{
5 Research Square

\section{Multiscale Feature Extraction and its Application in the Weld Seam Quality Prediction for Plasma Arc Welding}

Hao Dong

Shanghai Jiao Tong University

Cai Yan ( $\square$ ycai@sjtu.edu.cn )

Shanghai Jiao Tong University

Zihan Li

Shanghai Jiao Tong University

Xueming Hua

Shanghai Jiao Tong University

\section{Research Article}

Keywords: Multiscale feature extraction, Prediction model, Seam quality, Plasma arc welding (PAW), Model accuracy

Posted Date: July 26th, 2021

DOl: https://doi.org/10.21203/rs.3.rs-729452/v1

License: (c) (i) This work is licensed under a Creative Commons Attribution 4.0 International License. Read Full License

Version of Record: A version of this preprint was published at The International Journal of Advanced Manufacturing Technology on January 3rd, 2022. See the published version at https://doi.org/10.1007/s00170-021-08607-w. 


\title{
Multiscale Feature Extraction and its Application in the weld seam quality prediction for Plasma Arc Welding
}

\author{
Hao Dong ${ }^{\mathrm{a}}$, Yan Cai ${ }^{\mathrm{a}, \mathrm{b}}$ *, Zihan $\mathrm{Li}^{\mathrm{a}}$, Xueming Hua ${ }^{\mathrm{a}, \mathrm{b}}$ \\ a. Shanghai Key Laboratory of Materials Laser Processing and Modification, Shanghai Jiao Tong University, Shanghai, 200240, China \\ b. Collaborative Innovation Center for Advanced Ship and Deep-Sea Exploration, Shanghai, 200240, China \\ *The corresponding author, E-mail: ycai@ sjtu.edu.cn, ORCID identity: 0000-0002-7834-0414
}

\begin{abstract}
Weld seam quality prediction is important for intelligent robot welding. Current models with single scale feature extraction methods meet difficulty when facing complex physical instability in welding process. In this paper, a novel feature extraction method based on sliding multiscale windows is proposed to improve model accuracy and calculation speed. A group of windows with different width are established to extract multiscale information of complex objective. Windows slide throughout process and be synchronized on the timeline for feature correlation. Based on the feature vector extracted from multiscale-windows, Support vector machine (SVM) with radial basis function (RBF) kernel is used after signal denoising and dimension reduction by Primary components analysis (PCA). The best window width is determined by model training. The proposed method is used to predict seam quality for Plasma arc welding (PAW) in the field of shipbuilding. The results show that the model with multiscale feature extraction is helpful to improve prediction precision and recall ratio.
\end{abstract}

Keywords: Multiscale feature extraction; Prediction model; Seam quality; Plasma arc welding (PAW); Model accuracy

\section{Introduction}

Due to the complexity of welding process, it is difficult to build an intelligent stability monitoring system only with statistical or mathematical modeling methods. The methods based on Artificial Intelligence (AI) have an advantage in handling such high nonlinear problem. As reviewed by Oztemel and Gursev (2018), AI technology plays a vital role by minimizing human involvement, automatically and efficiently controlling the manufacturing process, and performing adaptive decision. Shevchik et al. (2020) proposed a method for real-time detection of laser welding process instabilities based on a deep artificial neural network. They used wavelet packet transform to extract features from the laser back reflected signal and acoustic emission signals. The quality classification ranged between $71 \%$ and $99 \%$, which proved the feasibility of deep learning method. Generally, there are abundant information in welding process signals. It is necessary to extract features for fetching relevant information and restraining interferences, and feature extraction becomes an essential part for AI-based methods. In recent studies, researchers developed many intelligent feature extraction methods for welding monitoring. You et al. (2015) used wavelet packet decomposition (WPD) method to extract features from optic signals in laser welding. They exerted primary component analysis (PCA) method to perform further refining for the features. A model used these features provided a mean accuracy of $80.17 \%$ on predicting defects like blowouts, humping and undercut. Zhang et al. (2019) performed WPD on optic signals during the process of laser welding. Statistical features 
were calculated in wavelet packet coefficients, and the features were used to build a convolutional neural network (CNN) model. Their model detected blowout, undercut, and humping defects with an accuracy of more than $93 \%$. In the studies, advanced signal analysis methods were used to extract feature information in welding signals without human involvement, and these methods performed well in many monitoring objects.

However, the requirements for model accuracy are constantly improving. To this end, the window width should be optimized in feature extraction process. At present, the window size of the evaluation process is normally determined by experts. Huang et al. (2017) determined the peak period ( 0.4 second) as calculation window to evaluate porosity defects for pulsed tungsten inert gas (TIG) welding of aluminum alloy. They computed a ratio of two spectral line intensities and their statistical parameters in every peak period of spectral signal. Huang et al. (2019a) observed laser welding process and found that the computed window with period of 0.002 second is suitable for predicting weld formation defects. In gas metal arc welding (GMAW) process, Huang et al. (2020) extracted features in the window with time length of 0.4096 second. The accuracy of their model for weld formation was $98.75 \%$. There are significant differences of window width among prediction models. This is due to the differences of welding principles, influencing factors and detection targets. For instance, in laser welding, Wang et al. (2012) studied the oscillation of plasma and found out the fluctuation period was around 450-600 $\mu \mathrm{s}$. Huang et al. (2019b) concluded in their study that the fluctuation frequency of plasma is between 1.5 and $3 \mathrm{kHz}$, for keyhole it is between 200 and $700 \mathrm{~Hz}$, and noise can be at the order of $10 \mathrm{kHz}$. Improper calculation window will lead to information loss, which will reduce the accuracy of models. Although manual determination is helpful, it increases the dependence of evaluation methods on experts. To this end, it is necessary to design a method to determine the most appropriate mode for window settings.

This paper aims to present a multiscale signal feature extraction method to extract information within different time scales synchronously. Signals are filtered using wavelet denoising. A group of sliding windows with different width are established. The multiscale windows are synchronized on the timeline for feature correlation. After windows initialization, SVM with RBF kernel, as well as dimension reduction by PCA, is used to establish prediction model. Through labeling and training, the optimum parameters of window width are determined. The proposed method was used in seam quality prediction for plasma arc welding (PAW) in the field of shipbuilding. Its performance is compared with single window models. The comparison results show that it is helpful to promote the precision and recall ratio of model.

\section{Method and procedure}

The core of proposed method is to extract information from complex objective with multiscale windows. The purpose is to improve the accuracy of prediction. The settings of windows are critical. To this end, the training based on a group of labeled data should be carried out for window optimization. Accordingly, the method is divided into two parts: training and prediction, as shown in Fig. 1. In the training part, there are three steps: (1) signal preprocessing, (2) feature extraction with multiscale windows, and (3) model training to determine the optimum window width. When the settings of windows are optimized, the prediction model is established. In the prediction part, the information extracted by multiscale windows is used as input vector of model to output prediction results. The multiscale feature extraction is helpful to obtain effective and adequate information, which is helpful to improve model accuracy. 


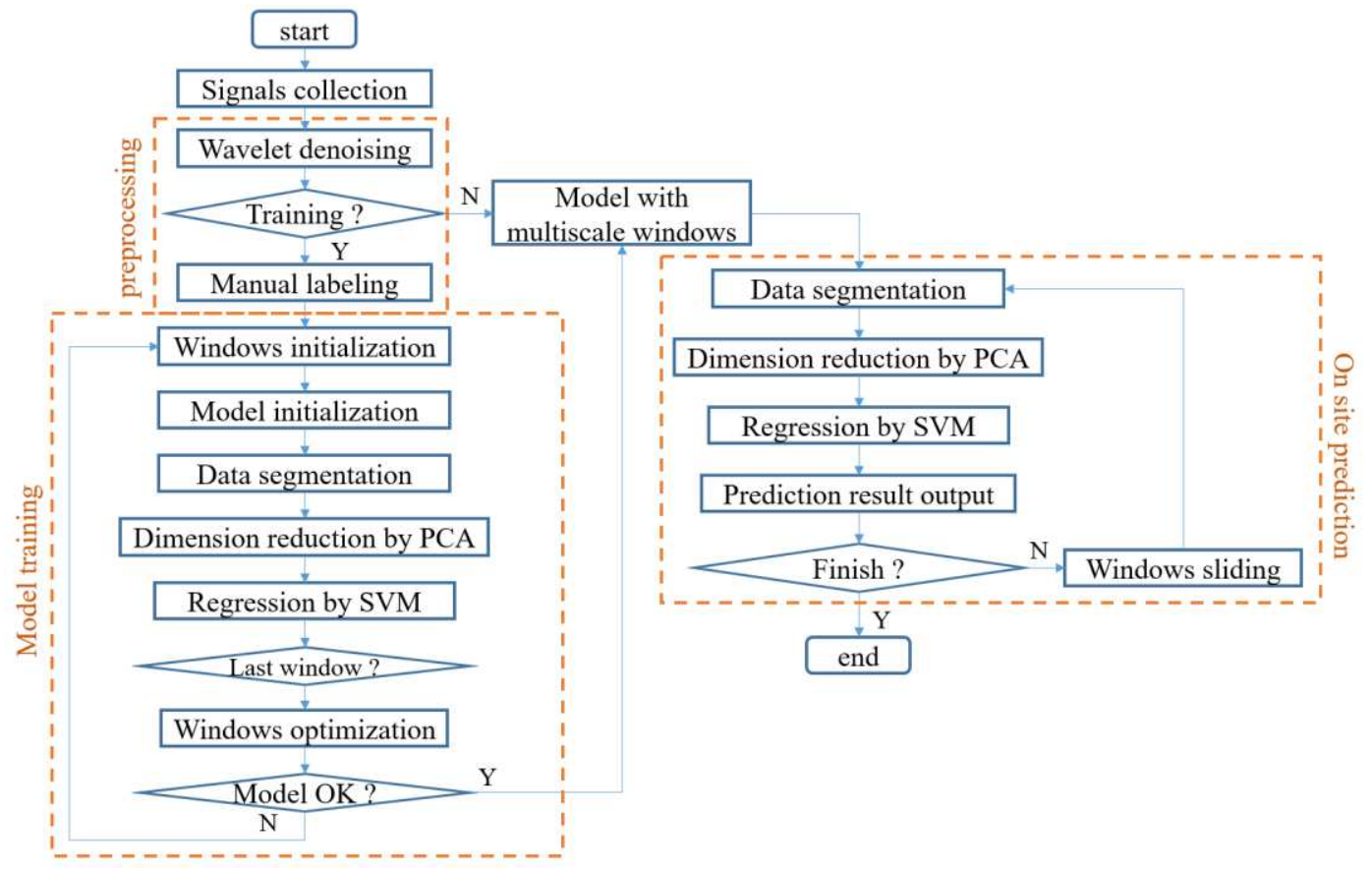

Fig. 1 The flow diagram of the proposed multiscale feature extraction method

\subsection{Signal denoising}

It is difficult to avoid noise in signals collected on site. The frequency of noise is usually high. When the signal-tonoise ratio is low, it is very unfavorable to the information extraction. The optimization of multiscale windows would be affected. To this end, signals are denoised by wavelet denoise method. The method is based on the principle of discrete wavelet transform (DWT). DWT is a widely used wavelet transform method which decomposes the signal into a series of components with different frequency ranges, developed by Mallat (1989). The main formula of DWT can be written as:

$$
\begin{gathered}
\boldsymbol{x}[m]=\frac{1}{\sqrt{N}} \sum_{k} W_{\varphi}\left[j_{0}, k\right] \varphi_{j_{0}, k}[m]+\frac{1}{\sqrt{N}} \sum_{j=j_{0}}^{\infty} \sum_{k} W_{\psi}[j, k] \psi_{j, k}[m], \quad m=0,1, \ldots, N-1 \\
W_{\varphi}\left[j_{0}, k\right]=\left(\boldsymbol{x}, \varphi_{j_{0}, k}\right)=\frac{1}{\sqrt{N}} \sum_{m=0}^{N-1} \boldsymbol{x}[m] \varphi_{j_{0}, k}[m], \\
W_{\psi}[j, k]=\left(\boldsymbol{x}, \psi_{j, k}\right)=\frac{1}{\sqrt{N}} \sum_{m=0}^{N-1} \boldsymbol{x}[m] \psi_{j, k}[m], \quad j>j_{0}
\end{gathered}
$$

where $j$ and $k$ are the parameters controlling the scale and shift of the basis wavelet functions $\varphi$ and scale functions $\psi$. $W_{\varphi}\left[j_{0}, k\right]$ and $W_{\psi}[j, k]$ are called the approximation coefficient and detail coefficient, respectively. The two coefficients stand for the low frequency and high frequency components of the signal. In wavelet denoise method, for each component from DWT, a threshold is set to filter the high frequency values. After filtering, the components are reconstructed into the denoised signal through a reverse process. 


\subsection{Multiscale windows}

After denoising, signals of complex process usually have different frequency components. Especially in the case of defects caused by process instability, the information usually comes from different frequency components. Take the welding process as an example, weld defects form due to the unstable welding process. Instability can be reflected in abnormal fluctuation of process signals. The frequency of fluctuation is affected by physical factors including materials, joint types, welding methods, environment factors etc. Even in a single welding process, the frequency of the fluctuation may change. Fig. 2(a) showed a filtered voltage signal collected in welding process, where fluctuation can be seen at the start period. The weld formation at initial part was poor because there is a big assembly gap. With the decrease of assembly gap, the welding process became stable in the second half of weld seam. Two sections of instable welding process are, section (1) and section (2), are used to investigate the effects of window width. Windows with different width are set for feature extraction from welding voltage signals, as shown in Fig. 2(b) and (c). When window width is $0.1 \mathrm{~s}$, it's difficult to extract valid information from section (1) since there is little change of voltage signal. Relatively, the information contained in section (2) is adequate. However, situation changes when the window width is increased to $2 \mathrm{~s}$. The amount of information in section (1) is appropriate, but there is superposition of different scale information in section (2). From this point of view, the window width is critical to feature extraction. Accordingly, a method that can extract features in different time scales should be developed to solve such problem.

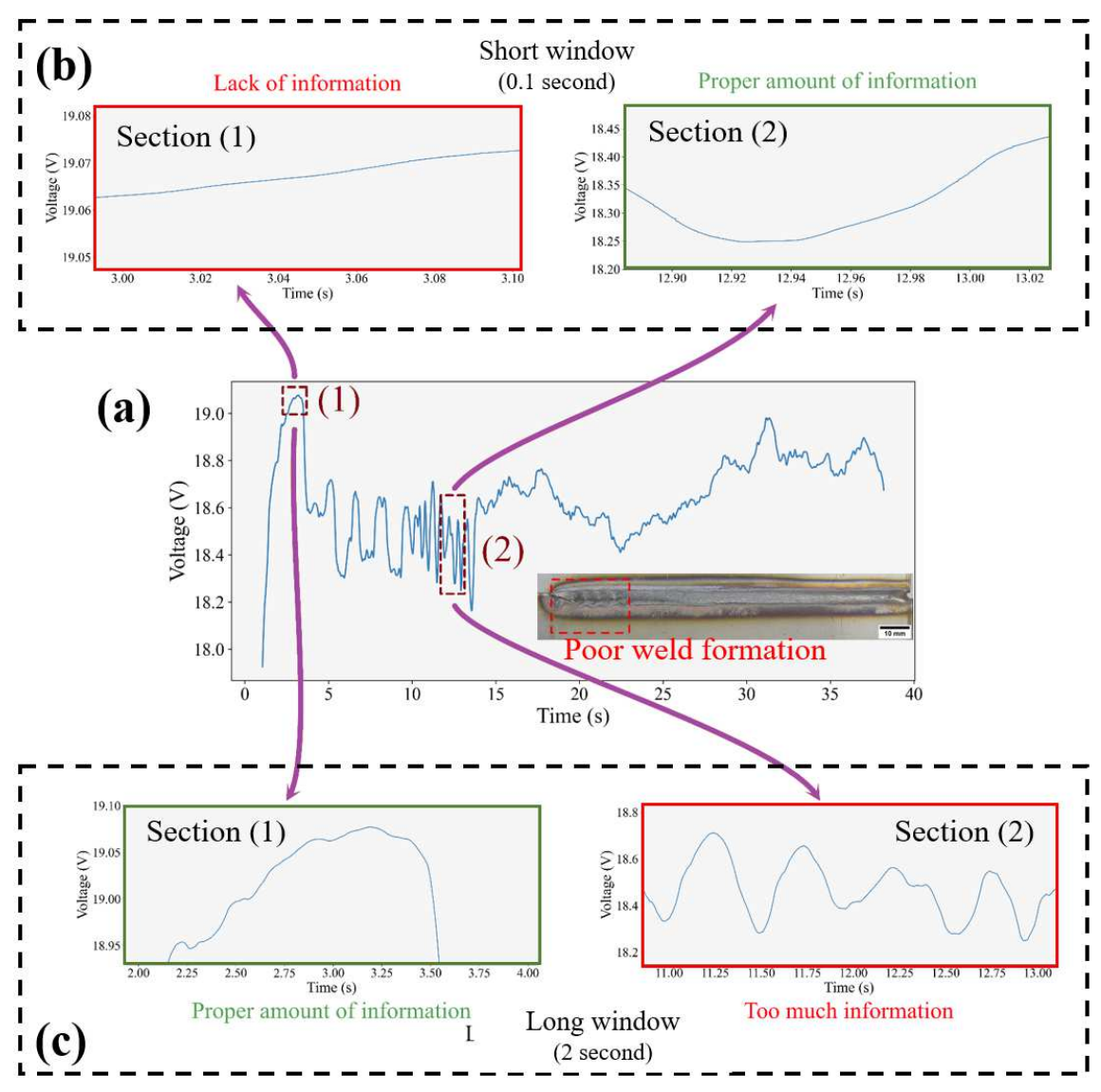

Fig. 2 An example describing the shortcoming of single scale models.

For objects with certain complexity, it is necessary to use multiple windows with different widths in time domain analysis. Here, a setting method of calculation windows is proposed. A group of windows with different width are 
established, and feature extraction is carried out inside windows. Normally, short windows focus on details, while long windows are capable to extract features that contain long-term information. Windows slide throughout process. In addition, the multiscale windows should be synchronized on the timeline for feature correlation.

Fig. 3 presented the setting method of multiscale windows. The initialization of window width needs basic cognition of the analyzed object. The requirement of expert knowledge is not necessary. Take three windows as an example, all windows are aligned at their end time. The moving distance between neighbored windows is set to be equal to the shortest window width. Before slide, a feature extraction is carried out inside every window. The features are used for model prediction. In this mode, there is no overlap for the shortest window. For other windows, there are some same data points in the two calculation processes. The sliding step length and calculation frequency are determined by the shortest window. At every iteration, the number of windows and their width kept constant. Through iterations, the width of windows is optimized by model training.

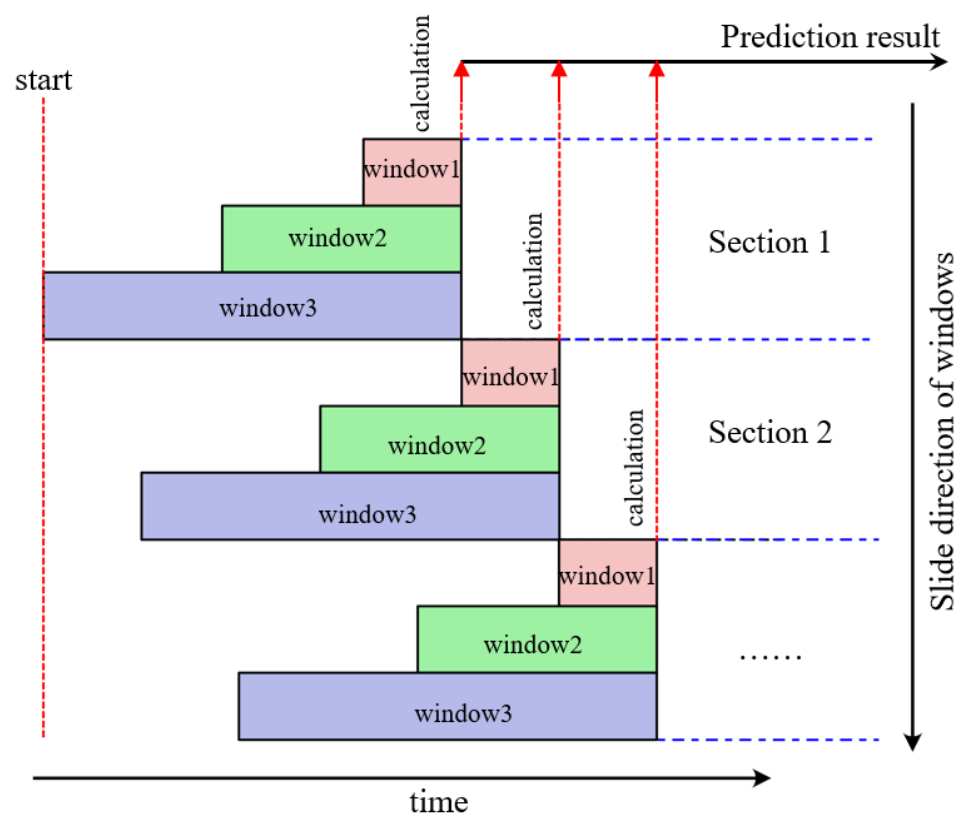

Fig. 3 Schematic of multiscale windows settings and sliding

\subsection{Modeling procedure}

Primary components analysis (PCA), proposed by Pearson (2010), is used to extract features in windows with different width. PCA is a widely used method of feature extraction and data dimension reduction. It transforms the original data to a new coordinate system where the greater variance by some projection of the data lies on the former coordinates (called primary components). The data matrix $\boldsymbol{V}$ is centered at first:

$$
\widehat{V}=\boldsymbol{V}-\overline{\boldsymbol{V}}
$$

where $\overline{\boldsymbol{V}}$ is the mean vector of the feature vectors in $\boldsymbol{V}$. The covariance matrix $\boldsymbol{C}$ of the data matrix is calculated by

$$
\boldsymbol{C}=\frac{1}{n} \widehat{\boldsymbol{V}} \widehat{\boldsymbol{V}}^{\mathrm{T}}
$$

The eigenvector $\boldsymbol{u}_{i}$ of the covariance matrix is obtained by solving the equation

$$
\lambda_{i}=\boldsymbol{C} \boldsymbol{u}_{i}, \quad i=1,2, \ldots, n
$$


where $\lambda_{i}$ is the $i$ th eigenvalue of $\boldsymbol{C}$, and $\lambda_{1} \leq \lambda_{2} \leq \cdots \leq \lambda_{n}$. Then the transformed feature matrix $\boldsymbol{S}$ can be calculated by

$$
\boldsymbol{S}=\boldsymbol{U} \widehat{\boldsymbol{V}},
$$

where each row contains a primary component, and the primary components are sorted in the order of importance. By choosing the number of rows to be used, the feature matrix's dimension can be reduced. The number of primary components is determined by the information contains in them, where the information ratio is often represented by variance ratio of the component. Here, the number of components is set to contain at least $90 \%$ of sample's variance.

Subsequently, the feature vectors with the same ending time are connected end to end to form a dataset that contains multiscale information. The new feature dataset can be used in machine learning models. To determine the optimum window size, a predict model is built and trained with the dataset with different window width. The dataset is divided into training set and test set. Here, support vector machine (SVM) with radial basis function (RBF) kernel is used as the machine learning model. SVM is a common machine learning model developed by Cortes and Vapnik (1995), which has been applied to various fields. The main formula of SVM can be expressed as:

$$
\begin{gathered}
\min _{\boldsymbol{w}, b, \xi} J(\boldsymbol{w})=\min _{\boldsymbol{w}, b, \xi} \frac{1}{2}\|\boldsymbol{w}\|^{2}+C \sum_{i=1}^{n} \xi_{i}, \\
\text { s.t. } \quad y_{i}\left(\boldsymbol{x}_{i}^{\mathrm{T}} \boldsymbol{w}+b\right) \geq 1-\xi_{i}, \\
\xi_{i} \geq 0, \quad i=1,2, \ldots, n
\end{gathered}
$$

where $y_{i}$ and $x_{i}$ are the label and the feature vector of the $i$ th sample, respectively, and $\xi$ is the distance between the inseparable points to the separating hyperplane. The hyper parameter $C$ is called regulation parameter which controls the strength of the penalty to the inseparable points. Kernel functions are used to map the input features to higher dimensional space to solve nonlinear problems. A frequently used kernel function is the RBF kernel, which is expressed as

$$
K\left(\boldsymbol{x}, \boldsymbol{x}^{\prime}\right)=\exp \left(-\gamma\left\|\boldsymbol{x}-\boldsymbol{x}^{\prime}\right\|^{2}\right),
$$

where $\boldsymbol{x}^{\prime}$ is the center of the kernel, and $\gamma$ is the shape parameter. The kernel function is used to replace input feature vector $\boldsymbol{x}$ in eq. (10) to solve nonlinear problems. The hyper parameters $C$ and $\gamma$ should be optimized, and this is usually done by using cross-validation method like grid search.

\section{Application and discussion}

\subsection{Application situation and objectives}

The proposed method was used to predict weld seam quality for plasma arc welding (PAW) in the field of shipbuilding. As shown in Fig. 4, the PAW system included an LHM-315 plasma welding machine, a TP-3 plasma torch, and a Yaskawa Shougang DX100 robot. A torch orifice with diameter of $1.6 \mathrm{~mm}$ was used in the experiment. The electrode diameter was $1.5 \mathrm{~mm}$, and the electrode setback was $3 \mathrm{~mm}$. The electrode tip angle was $20^{\circ}$. Pure argon was chosen as plasma and shielding gas, and the gas flow rate was $12 \mathrm{~L} / \mathrm{min}$. The welding current was $40 \mathrm{~A}$, and the welding speed was set to 20 $\mathrm{cm} / \mathrm{min}$. The welded material was 304L stainless steel. The thickness of the plates was $1.2 \mathrm{~mm}$, and the joint type was lap joint. Hall sensors were used to collect arc current and welding voltage signals, and the sampling rate was $20 \mathrm{kHz}$. 


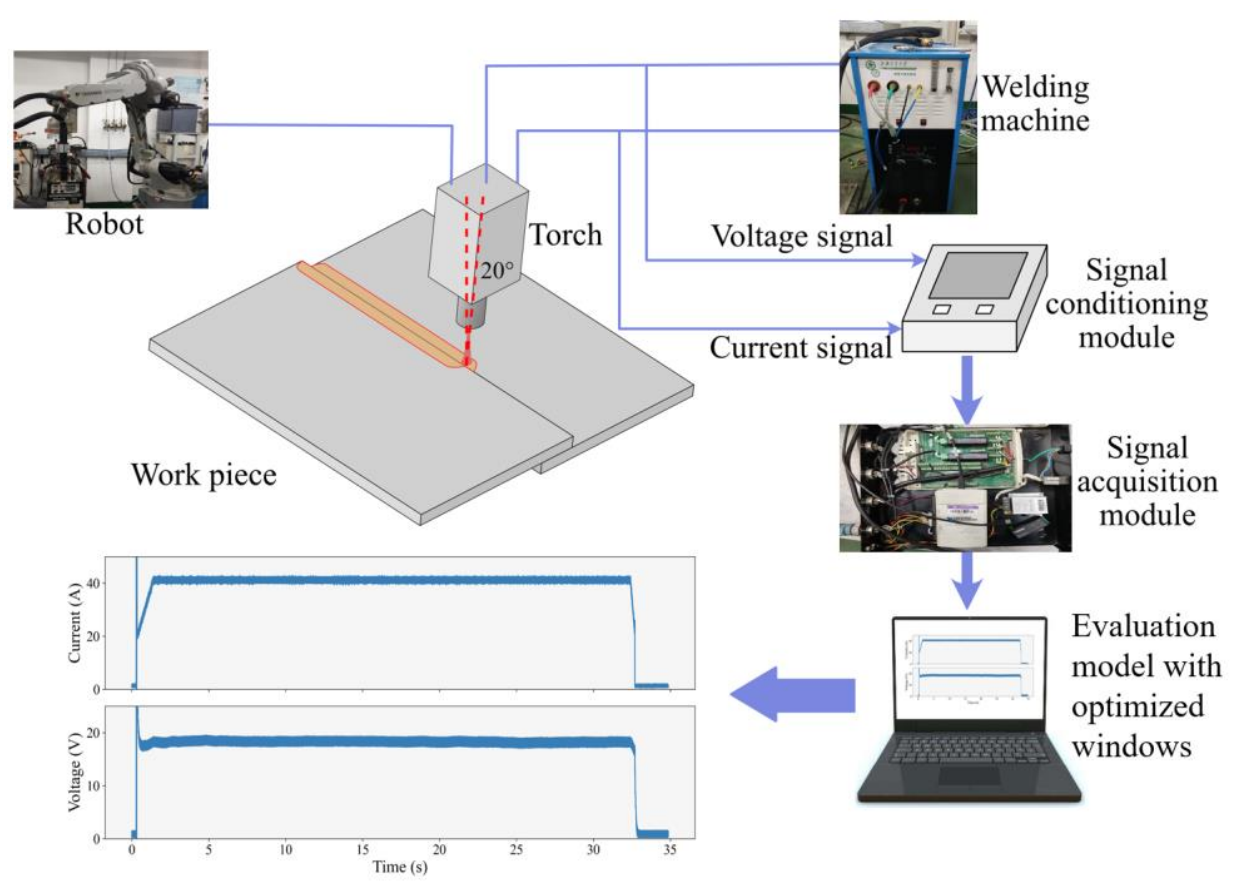

Fig. 4 PAW welding and signal acquisition system.

As base line, the distance between torch and workpiece in group 1\# was constant at $2 \mathrm{~mm}$ to keep plasma stable. As shown in Fig. 5, unstable welding conditions were created by changing the torch height. In group 2\#, torch was lifted $1 \mathrm{~mm}$ at the middle of welding process. On the contrary, torch height in group 3\# was reduced to $1 \mathrm{~mm}$ during welding process. In group 4\#, a spacer was used to increase the distance between torch and workpiece continuously. As results, the fluctuation of torch height led to unqualified weld formation, as shown in Fig. 6. Here, the current and voltage of plasma arc are collected as input signals of model. The surface quality of welded seam was the prediction target of model. According to surface defects and seam width uniformity, the weld process was divided into two types. In Fig. 6, the zones with poor formation are marked in red, which should be predicted by model in the process of robot welding. The remaining zones, as well as their corresponding welding processes are considered as qualified. The target of the proposed model is to predict the seam quality based on electrical signals and present evaluation results on line.
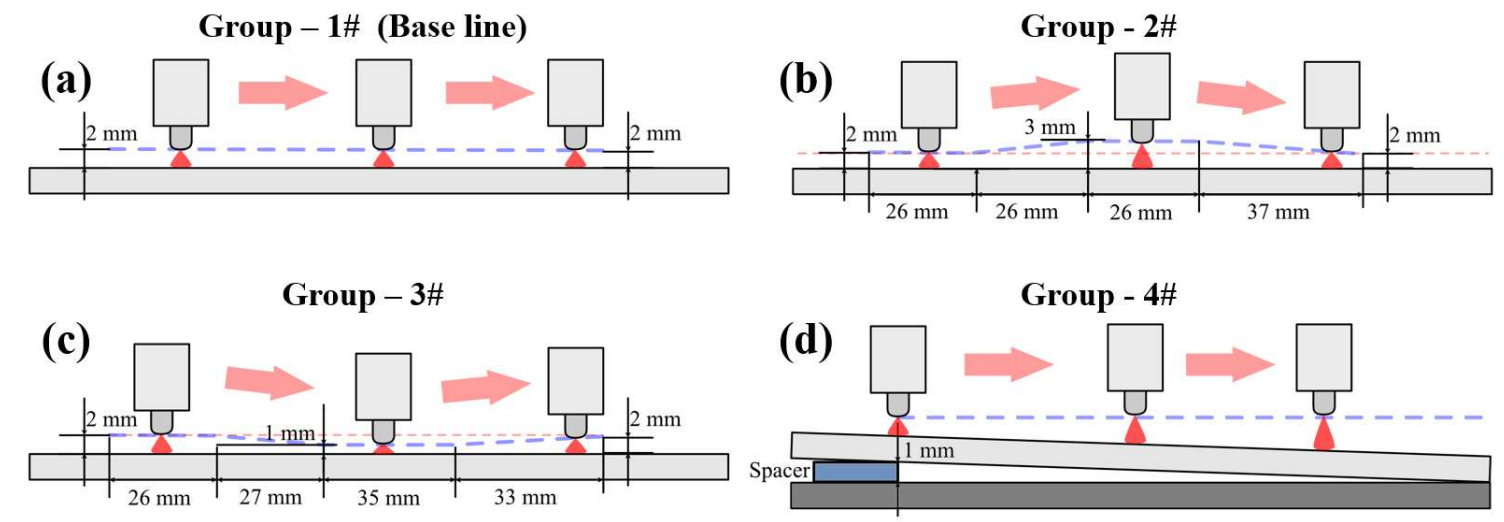

Fig. 5 Experiment settings: (a) base line group; (b) group 1\#; (c) group 2\#; (d) group $3 \#$. 

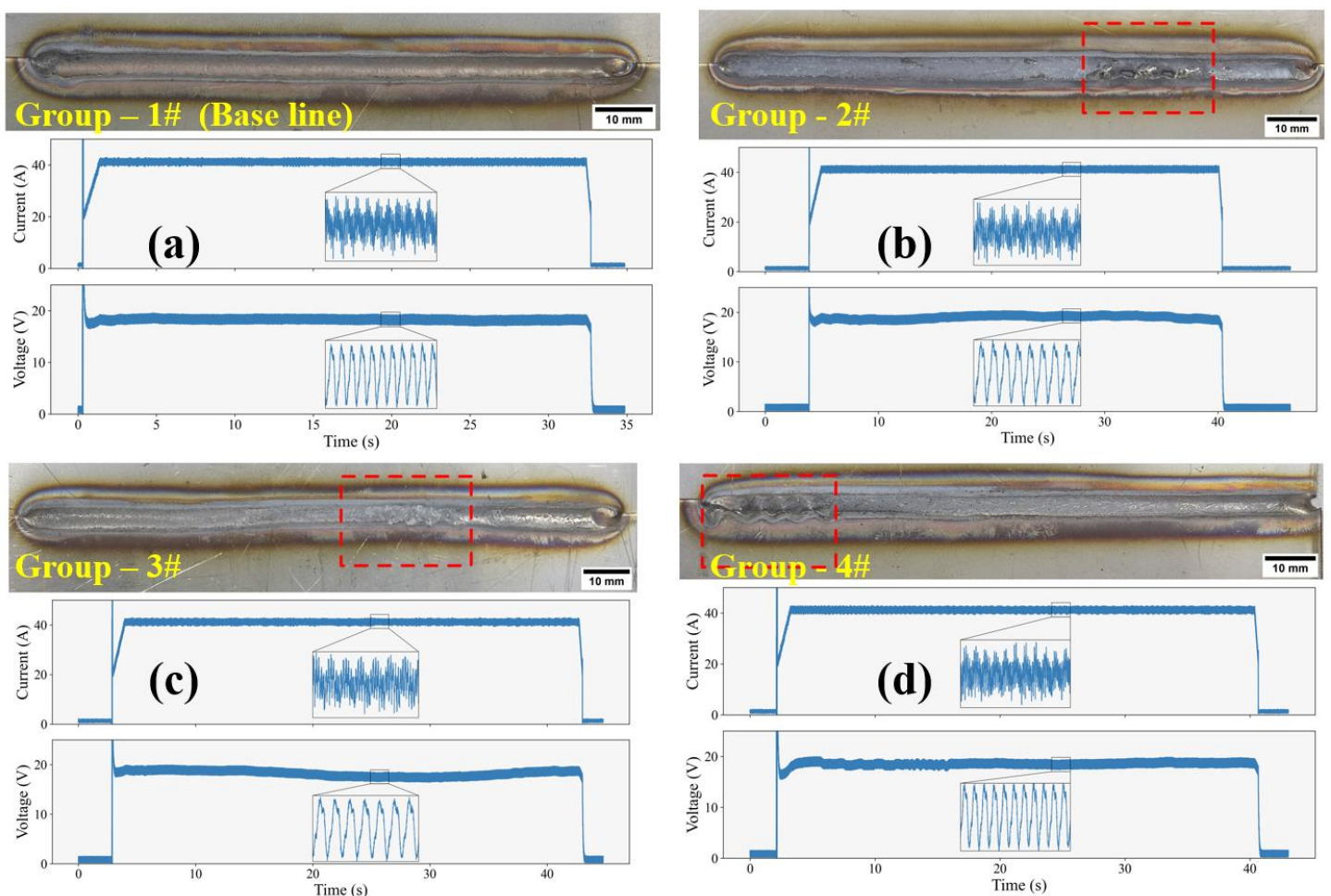

Fig. 6 The welded seams (unstable part marked in red) and corresponding signal waveforms: (a) group 1\#; (b) group 2\#; (c) group 3\#; (d) group 4\#.

\subsection{Modeling and windows optimization}

During robot welding, electrical signals of plasma arc are easy to collect on site. It provides conditions for the industrial application of model. However, there is a lot of noise in signals, which is disadvantageous to the subsequent feature extraction. To this end, the denoising of raw signals are carried out firstly. The wavelet denoise method is used to remove the high-frequency noise in the signal and keep the fluctuation of the signal as much as possible. Here, the denoise threshold in this experiment was set as follows:

$$
\text { Thresold }=\sqrt{2 \ln (\operatorname{length}(x))},
$$

where length $(\boldsymbol{x})$ denotes the length of the signal. The threshold type used was soft threshold. The wavelet type was set to ' $\mathrm{db} 4$ ' wavelet, and the decomposition level was set to no more than 11. Compare to the raw signals presented in Fig. 6, the denoised waveform present in Fig. 7 has less high frequency noise, but the fluctuation trend and local details are preserved. 


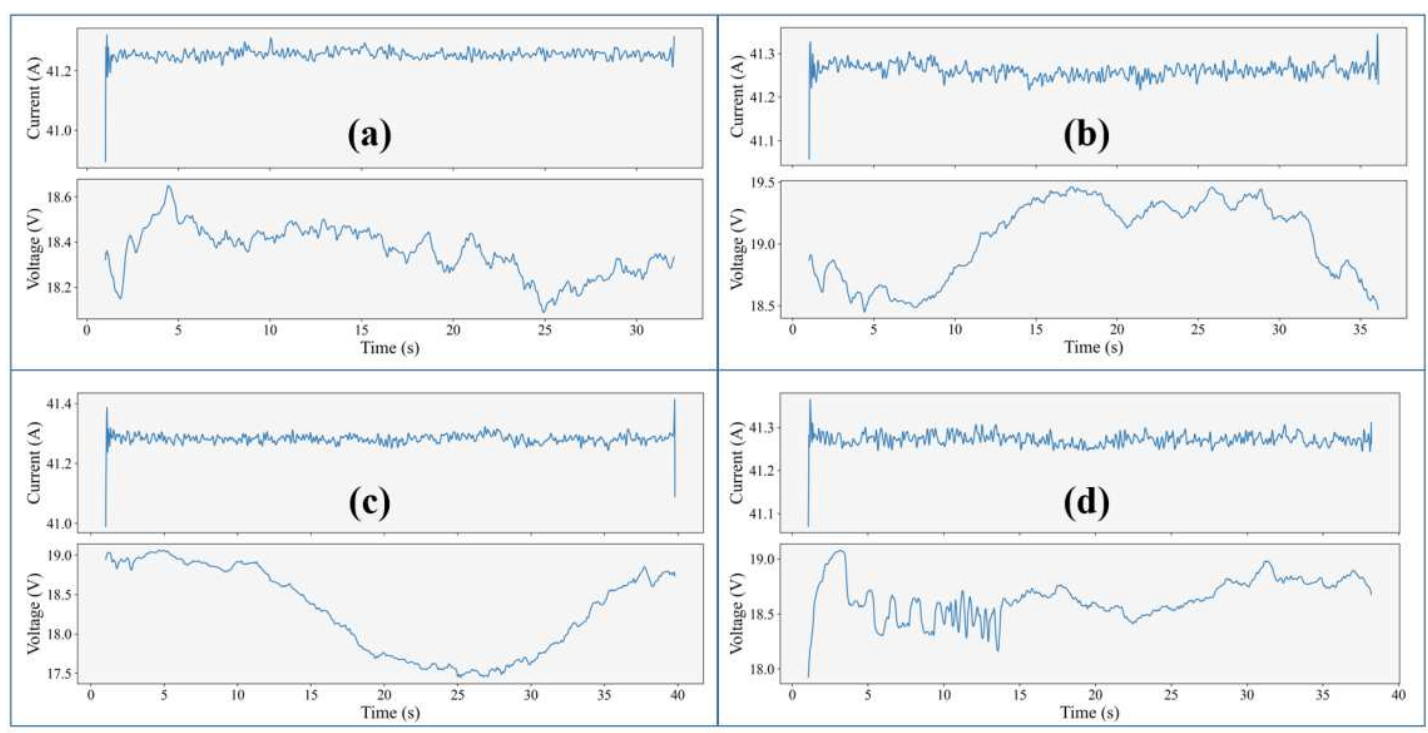

Fig. 7 Signals after denoising: (a) group 1\#; (b) group 2\#; (c) group 3\#; (d) group 4\#.

After dimension reduction based on PCA, an SVM model with RBF kernel was established. The number of principal components is 3 . The hyper parameters $C$ and $\gamma$ of SVM model were optimized using grid search cross-validation method. The candidate values of the hyper parameters are set to $\{0.1,1,10,100,1000\}$. Because the result of hyper parameter optimization may differ according to the choice of segment lengths, the specific optimization results are not discussed in this paper, and the final model always used the best hyper parameters to produce the outputs.

Here, the weld seam quality is the prediction target, and it is divided into poor formation and qualified formation. Two windows are set for feature extraction. The short window is designed for local and short-term disturbances, while the long one is to collect long-term fluctuation or trend in welding process. According to Wang and Chen (2002), for PAW process, fluctuation frequency could lie in a range of $0.5 \mathrm{~Hz}$ to $1 \mathrm{kHz}$, and the frequency was found widely distributed within 250 $\mathrm{Hz}$. Therefore, the range of segment lengths was set between $0.001 \mathrm{~s}$ and $2 \mathrm{~s}$. The short window width was chosen in the list of $\{0.001 \mathrm{~s}, 0.005 \mathrm{~s}, 0.01 \mathrm{~s}, 0.05 \mathrm{~s}, 0.1 \mathrm{~s}\}$, and the long window width in the list of $\{0.1 s, 0.5 s, 1.0 s, 1.5 s, 2.0 s\}$. Window width, as well as their match, is the optimization objective of the proposed model.

Orthogonal experiments were performed for this purpose. $70 \%$ of the dataset was used as training set, and the remaining $30 \%$ was used as test set.

During training, 5-fold cross-validation method was used to reduce over fitting. Accuracy for test set and total computation time cost were used to evaluate the performance of the model. The accuracy was defined as:

$$
A_{t}=\frac{n_{c}}{n}
$$

where $n_{c}$ and $n$ represents the number of corrected classified samples and the total number of samples in the test set, respectively. Computation time was the total time cost of data segmentation, feature extraction, model training, and model testing.

According to the results presented in Fig. 8(a), the model accuracy was significantly affected by window widths. The window settings located on the upper left corner has high accuracy. It indicates that the longer the long window, the shorter 
the short window, the higher the accuracy of the model. As the width of short window decreases, the accuracy of model increases rapidly. It proves that detail information contributes a lot for prediction. With the increment of the long window width, the performance of model slightly increases. It reflects the relationship between the weld formation and the longterm fluctuation trend of welding process. The accuracy drops when the width of short window and long window are close. It could be attributed to the information loss on both local disturbances and long-term trend. From the point of model accuracy, the two-window model has a wide range of parameter selection. It indicates that modeling with multiscale windows is helpful to improve model accuracy. As shown in Fig. 8(b), the computation time cost mainly depends on the width of short window. The shorter the window, the more the number of windows. It results in a large amount of calculation.
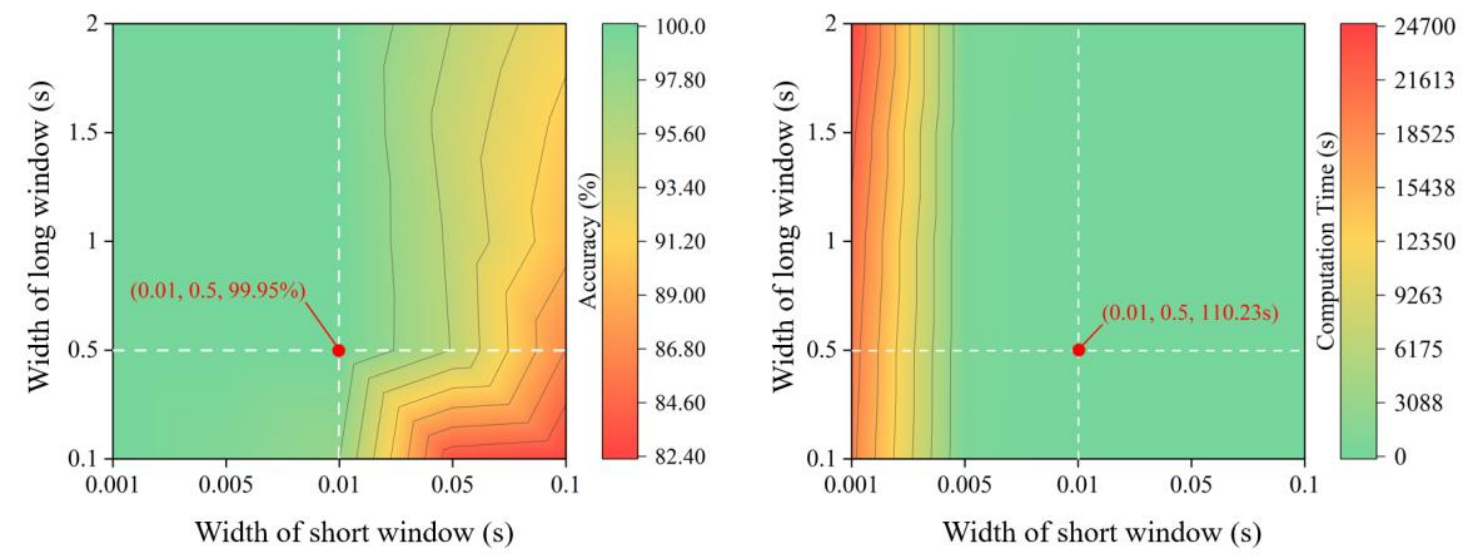

Fig. 8 Model performance with different windows (location of the optimized model is marked as red point): (a) model accuracy; (b) computation time.

During the optimization of windows, the model accuracy and calculation time should be balanced. In Fig. 8(a), the accuracy of model has the best performance when the short window width is less than $0.01 \mathrm{~s}$ and the long window width is larger than 0.5 s. In Fig. 8(b), the computation cost increases significantly when short window width is less than 0.005 $\mathrm{s}$. Therefore, the best choice in this case is $0.01 \mathrm{~s}$ for short window and $0.5 \mathrm{~s}$ for long window. The optimized matching between short window and long window is marked as a red point in Fig. 8.

\subsection{Model performance}

The model with optimized windows, namely $0.01 \mathrm{~s}$ short window and $0.5 \mathrm{~s}$ long window, is established to predict seam quality for PAW. The prediction result of the model is compared with models using a single window. The window width of single window model is $0.005 \mathrm{~s}, 0.01 \mathrm{~s}, 0.05 \mathrm{~s}, 0.1 \mathrm{~s}, 0.5 \mathrm{~s}, 1 \mathrm{~s}$, and $2 \mathrm{~s}$. The precision and recall for both qualified formation and poor formation are calculated as following:

$$
\begin{gathered}
\text { Precision }=\frac{T P}{T P+F P}, \\
\text { Recall }=\frac{T P}{T P+F N},
\end{gathered}
$$

where TP represents the number of correctly classified positive samples, $F P$ denotes the number of wrongly classified positive samples, and $F N$ means the number of wrongly classified negative samples. Specially, if the number of classified samples is 0 , the precision is also set to 0 . The result is presented in Table 1 . 
Table 1. Performance in test set of prediction models with different windows

\begin{tabular}{lcccc}
\hline \multirow{2}{*}{ Model } & \multicolumn{2}{c}{ Qualified formation } & \multicolumn{2}{c}{ Poor formation } \\
\cline { 2 - 5 } & Precision & Recall & Precision & Recall \\
\hline Single window (0.001 s) & $96.948 \%$ & $96.195 \%$ & $82.015 \%$ & $85.144 \%$ \\
Single window (0.005 s) & $97.649 \%$ & $91.595 \%$ & $68.677 \%$ & $89.313 \%$ \\
Single window (0.01 s) & $93.464 \%$ & $92.505 \%$ & $64.555 \%$ & $67.847 \%$ \\
Single window (0.05 s) & $89.910 \%$ & $85.576 \%$ & $45.304 \%$ & $55.034 \%$ \\
Single window (0.1 s) & $92.150 \%$ & $76.705 \%$ & $37.879 \%$ & $68.493 \%$ \\
Single window (0.5 s) & $88.235 \%$ & $92.308 \%$ & $50.000 \%$ & $38.462 \%$ \\
Single window (1 s) & $80.488 \%$ & $100.00 \%$ & $0.000 \%$ & $0.000 \%$ \\
Single window (2 s) & $86.364 \%$ & $100.00 \%$ & $0.000 \%$ & $0.000 \%$ \\
Multiscale windows & $99.943 \%$ & $99.986 \%$ & $99.437 \%$ & $99.718 \%$ \\
\hline
\end{tabular}

The result shows that the model using multiscale windows performs better than models with single window, both in the precision and recall of poor formation. The performance of single-window models varies significantly with the window width. Generally, the smaller segment length results in a better performance for the classification of poor formation areas. But this improvement is at the cost of computing time. The accuracy of the multiscale-window model is better than all single-window models. It proves that the proposed modeling method is helpful to improve the prediction accuracy of seam quality for PAW. Fig. 9 shows the prediction results in test set, where most samples are in good agreement with their actual seam states.

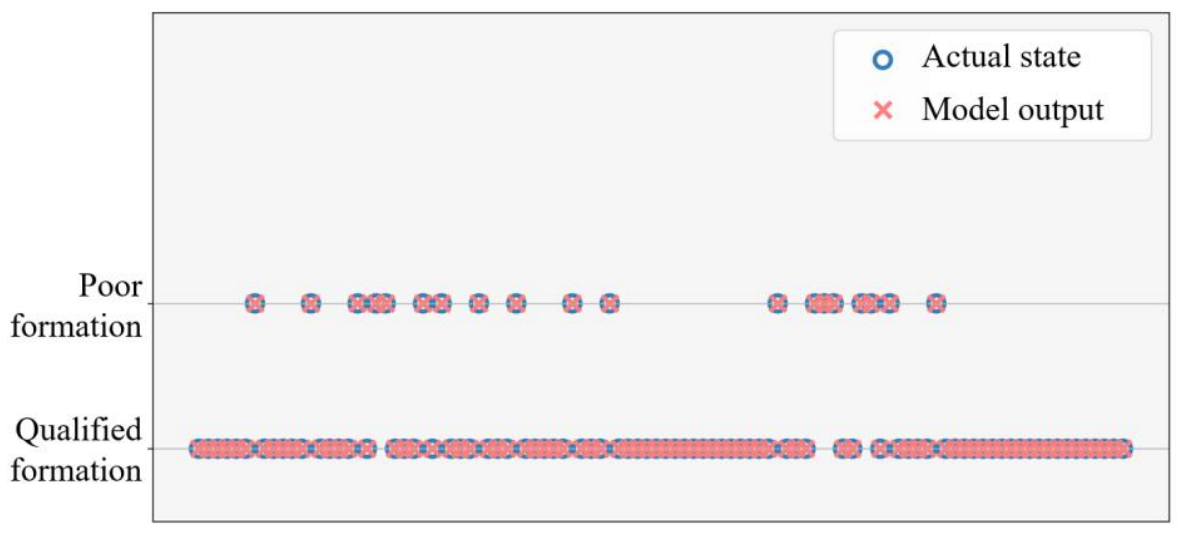

Fig. 9 Prediciton results in test set by proposed model

The model training was performed on a computer with a high-performance CPU of Intel Xeon E5-2470 (2.30 GHz with 16 cores and 32 threads). For industrial application, the trained model working on a CPU of Intel i5-8300H $(2.30 \mathrm{GHz}$ with 4 cores and 8 threads) is used to predict weld seam quality. Table 2 shows the average time cost for each steps of onsite prediction. The total computation time for one weld is 3.612 millisecond, less than the time period (10 millisecond) of a welding process. In terms of accuracy and computation time, the proposed model has good engineering application ability. Currently, the most time-consuming step is the signal preprocessing, and mainly comes from the wavelet denoising. In future application, a physical filter circuit would be added to the acquisition equipment to reduce or remove the need for 
digital filtering, by which the model's computation time can be greatly saved.

Table 2. Average time cost of steps in prediction process

\begin{tabular}{ccccc}
\hline Step name & Preprocessing & PCA feature extraction & SVM classification & Total \\
\hline Time cost (millisecond) & 3.225 & 0.363 & 0.024 & 3.612 \\
\hline
\end{tabular}

\section{Conclusions}

A feature extraction method based on multiscale windows was proposed to improve the accuracy and response speed of evaluation model. Its application in seam quality prediction proves its applicability in the field of shipbuilding. The intelligent level of robot welding is improved. The conclusions are as follows:

(1) Signals in complex process contain information in a wide frequency range. It's difficult for model with single window to extract effective information rapidly. Modeling with multiscale windows is helpful to extract information of both momentary disturbances and long-term fluctuation trend. The optimization of window width is the dominant factor for model accuracy.

(2) To establish multiscale-window model, SVM with RBF kernel is used after signal denoising and dimension reduction by PCA. The windows with different width are aligned to time stamp. For each type of window, the shift distance between neighbor windows is equal to its window width. The optimization of window width is carried out aiming at both model accuracy and calculation time.

(3) A model with two scale windows is established to predict seam quality of PAW in the field of shipbuilding. The width of short window is 0.01 second, while the width of long window is 0.5 second. The prediction precision is $99.943 \%$ and recall ratio is $99.986 \%$. Its calculation time is 3.612 millisecond. The comprehensive performance of prediction model is significantly improved by multiscale feature extraction method.

\section{Funding}

This work was financial supported by the Ministry of Industry and Information Technology of China under the project of "Research and Application of Materials for Membrane-Type (MARK III Type) Containment System".

\section{Conflicts of interest}

The authors declare that they have no competing interests.

\section{Availability of data and material}

The raw data and processed data required to reproduce these findings are available to download from https://data.mendeley.com/datasets/d78n73x35d/draft?a=ce94c66d-3e31-4e64-a689-0e645303c57d.

\section{Code availability}

Not applicable. 


\section{Ethics approval}

Not applicable.

\section{Consent to participate}

Not applicable.

\section{Consent for publication}

Not applicable.

\section{Authors Contributions}

Hao Dong was in charge of the evaluation modeling, parameter optimization, and also took part in paper writing. Yan Cai was in charge of research design, investigation and paper writing. Zihan Li was in charge of welding experiments. Xueming Hua took part in the research design and results discussion.

\section{Reference}

Cortes, C., Vapnik, V., 1995. Support-vector Networks. Machine Learning 20, 273-297.

Huang, Y., Wu, D., Zhang, Z., Chen, H., Chen, S., 2017. EMD-based pulsed TIG welding process porosity defect detection and defect diagnosis using GA-SVM. Journal of Materials Processing Technology 239, 92-102.

Huang, Y., Hou, S., Xu, S., Zhao, S., Yang, L., Zhang, Z., 2019a. EMD- PNN based welding defects detection using laserinduced plasma electrical signals. Journal of Manufacturing Processes 45, 642-651.

Huang, Y., Yang, D., Wang, K., Wang, L., Fan, J., 2020. A quality diagnosis method of GMAW based on improved empirical mode decomposition and extreme learning machine. Journal of Manufacturing Processes 54, 120-128.

Huang, Y., Xu, S., Yang, L., Zhao, S., liu, Y., Shi, Y., 2019b. Defect detection during laser welding using electrical signals and high-speed photography. Journal of Materials Processing Technology 271, 394-403.

Mallat, S.G., 1989. A theory for multiresolution signal decomposition: the wavelet representation. IEEE Transactions on Pattern Analysis and Machine Intelligence 11, 674-693.

Oztemel, E., Gursev, S., 2018. Literature review of Industry 4.0 and related technologies. Journal of Intelligent Manufacturing 31, 127-182.

Pearson, K., 2010. LIII. On lines and planes of closest fit to systems of points in space. The London, Edinburgh, and Dublin Philosophical Magazine and Journal of Science 2, 559-572.

Shevchik, S., Le-Quang, T., Meylan, B., Farahani, F.V., Olbinado, M.P., Rack, A., Masinelli, G., Leinenbach, C., Wasmer, K., 2020. Supervised deep learning for real-time quality monitoring of laser welding with X-ray radiographic guidance. Sci Rep 10, 3389.

You, D., Gao, X., Katayama, S., 2015. WPD-PCA-Based Laser Welding Process Monitoring and Defects Diagnosis by Using FNN and SVM. IEEE Transactions on Industrial Electronics 62, 628-636.

Wang, J., Wang, C., Meng, X., Hu, X., Yu, Y., Yu, S., 2012. Study on the periodic oscillation of plasma/vapour induced during high power fibre laser penetration welding. Optics \& Laser Technology 44, 67-70.

Wang, Y., Chen, Q., 2002. On-line quality monitoring in plasma-arc welding. Journal of Materials Processing Technology 120, 270-274.

Zhang, Y., You, D., Gao, X., Zhang, N., Gao, P.P., 2019. Welding defects detection based on deep learning with multiple optical sensors during disk laser welding of thick plates. Journal of Manufacturing Systems 51, 87-94. 